\title{
Nickel nanowires induced and reactive oxygen species mediated apoptosis in human pancreatic adenocarcinoma cells
}

This article was published in the following Dove Press journal:

International Journal of Nanomedicine

16 July 2011

Number of times this article has been viewed

\author{
Md. Zakir Hossain' \\ Maurice G Kleve 2 \\ 'Applied Biosciences \\ (Bionanotechnology Research), \\ Department of Applied Science, \\ ${ }^{2}$ Molecular Biotechnology \\ and Microscopy Laboratory, \\ Department of Biology, \\ University of Arkansas \\ at Little Rock, Little Rock, \\ Arkansas, USA
}

Background: The ability to evade apoptosis is one of the key properties of cancer. The apoptogenic effect of nickel nanowires (Ni NWs) on cancer cell lines has never been adequately addressed. Due to the unique physicochemical characteristics of Ni NWs, we envision the development of a novel anticancer therapeutics specifically for pancreatic cancer. Thus, we investigated whether Ni NWs induce ROS-mediated apoptosis in human pancreatic adenocarcinoma (Panc-1) cells.

Methods: In this study Ni NWs were fabricated using the electrodeposition method. Synthesized Ni NWs were physically characterized by energy dispersive X-ray analysis, UV-Vis spectroscopy of NanoDrop 2000 (UV-Vis), magnetization study, scanning electron microscopy, and transmission electron microscopy. Assessment of morphological apoptotic characteristics by phase contrast microscopy (PCM), Ni-NWs-induced apoptosis staining with ethidium bromide (EB) and acridine orange (AO) followed by fluorescence microscopy (FM) was performed. For molecular biological and biochemical characterization, Panc-1 cell culture and cytotoxic effect of Ni NWs were determined by using 3-(4, 5-dimethylthiazol-2-yl)-2, 5-diphenyl tetrazolium bromide (MTT) assay. Quantitative apoptosis was analyzed by flow cytometry staining with propidium iodide through cell cycle arrest and generation of ROS using $2^{\prime}, 7^{\prime}$-dichlorofluorescein diacetate fluorescence intensity. In all experiments, Panc-1 cancer cells without any treatment were used as the negative controls.

Results: The intracellular uptake of Ni NWs through endocytosis by Panc-1 cells was observed by PCM. EB and AO staining of FM and MTT assay qualitatively and quantitatively confirmed the extent of apoptosis. Flow cytometric cell cycle arrest and ROS generation indicated Ni NWs as inducers of apoptotic cell death.

Conclusion: We investigated the role of Ni NWs as inducers of ROS-mediated apoptosis in Panc-1 cells. These results suggested that Ni NWs could be an effective apoptotic agent for Panc-1 cells and have good potential for further research into a clinical treatment selective for pancreatic cancer.

Keywords: Ni NWs, apoptosis, Panc-1, MTT assay, flow cytometry, cell cycle arrest, ROS

\section{Introduction}

Pancreatic cancer has the highest fatality rates of all cancers; the 5-year survival rate is less than $5 \%$ due to intrinsic resistance of radiotherapy, immunotherapy, and chemotherapy. ${ }^{1}$ Current pancreatic cancer treatment involves surgery, radiation, and drugs alone or in combination, which temporarily relieves symptoms, increases survival rates, and occasionally cures. ${ }^{1}$ Apoptosis is derived from Greek "apo" meaning away from and "ptosis" meaning to drop or to fall. ${ }^{2}$ Apoptosis is characterized by specific morphological features and biochemical cascades. ${ }^{3}$ Morphological changes during apoptosis
Correspondence: Md. Zakir Hossain University of Arkansas at Little Rock, Biology, FH-406, 280I South University Avenue, Little Rock, AR-72204, USA

$\mathrm{Tel}+\mathrm{I} 5015693510$

Fax + I $50|569327|$

Email mxhossain I@ualr.edu 
include plasma membrane blebbing, initial cell shrinkage, chromatin condensation, and formation of apoptotic bodies. ${ }^{4}$ However, the organelles remain intact and healthy looking at the very late stage of death through continuous metabolic activity for some considerable time. ${ }^{2}$ Cellular reactive oxygen species (ROS) are essential to cell survival. Additionally, ROS have important functions in pro-apoptotic pathways. ${ }^{4}$ ROS are generated through nickel exposure in certain cell types and thought to be a key step in nickel-induced apoptosis due to an apoptotic response. Therefore, cancer cells may escape potentially carcinogenic ability. 5,6

An ideal anticancer drug is expected to kill or inactivate cancer cells without causing excessive damage to normal cells. ${ }^{7}$ These criteria are achievable only by apoptosis induction in cancerous cells because the lifespan of both normal and cancerous cells are significantly affected by the rate of apoptosis. ${ }^{8}$ Thus, modulating or inducing apoptosis might be helpful in cancer therapy or preventions., ${ }^{79}$ Advanced nanomaterialbased drugs may offer the possibility of new and intriguing opportunities to eliminate the toxicity in human cancer cells.

Nickel (Ni) is a naturally occurring transition metal and trace element which is an essential nutrient for human and higher animals. ${ }^{10} \mathrm{~A}$ possible health benefit of nickel includes optimal growth, healthy skin and bone structure. ${ }^{11}$ However, nickel is a potent human carcinogen in large quantities with low mutational activity. The most carcinogenic ni-compounds are those that do not induce apoptosis. ${ }^{12} \mathrm{Ni}$-induced apoptosis was reported on Chinese hamster ovary (CHO) cells, T-cell hybridoma, Jurkat cells, and mouse epidermal JB-6 cells. ${ }^{5}$

By definition, a nanowire (NW) is a nanostructure that has a diameter restricted to tens of nanometers $\left(10^{-9} \mathrm{~m}\right)$ or less and an unconstrained length which shows an aspect ratio (length-to-width) of $\geq 1000$ higher. Nanowires (NWs) have numerous interesting properties that are not exhibited in bulk or 3-D materials. This is true because in nanowires, electrons are laterally quantum confined and occupied energy levels are different from the continuum of energy levels or bands of bulk substrates. The NWs have advantages over the same nanoparticles (NPs) in terms of geometrical anisotrophy, increased surface to volume ratio, and dipolar magnetic properties linked to the NW shape. ${ }^{10}$

Choi et al ${ }^{12}$ described Ni NWs are not toxic to cells and Guo et $\mathrm{al}^{13}$ reported that Ni NWs are not immediately toxic to cells and chronic exposure is needed for them to act as carcinogens. ${ }^{10,14} \mathrm{Ni}$ NWs can easily be internalized by cancer cells due to $\mathrm{Ni}^{+2}$ ions which are also present in metalloproteins. ${ }^{10}$ Due to this property, it is possible that Ni NWs can also inhibit the proliferation of certain cancer cells, ${ }^{14}$ thus implying their great potential in cancer biomedical and chemotherapeutic applications. Apoptosis suppression is a hallmark of human cancer. ${ }^{15}$ Currently apoptotic cell death inducers and regulators are considered to have significant potential for cancer therapy which is a desired goal of many cancer treatments. ${ }^{15}$ Though some nickel (II)-induced apoptosis has been reported recently, ${ }_{5,8}$ the findings do not represent the effects of Ni NWs on the pancreatic adenocarcinoma cells. Since nickel has the ability to induce apoptosis through generation of reactive oxygen species (ROS), ${ }^{5} \mathrm{Ni}$ NWs could be used to fight against cancer through tumor cell death induction.

Internalization of Ni NWs with cell cytoplasm may lead to internal reorganization of lysozomes and metalloproteins. ${ }^{16}$ To the best of our knowledge this study is the first to delineate that Ni NWs induce cell cycle arrest, increase percentage of sub- $\mathrm{G}_{1}$ fractions, and trigger ROS generation in Panc-1 cells. These are the key characteristics for accomplishing apoptosis. ${ }^{17}$ In close agreement with previous studies, ${ }^{10,12,14,16}$ our in vitro studies showed that the treatment of pancreatic cancer Panc-1 cells with Ni NWs resulted in a concentration and time dependent induction of apoptosis. Therefore, Ni NWs induced apoptosis shows promising potential as an innovative approach to pancreatic cancer therapy.

\section{Materials and methods Materials}

Whatman Anodisc alumina filters (diameter of $25 \mathrm{~mm}$ with polypropylene support rings and 200 nanometer pores) were purchased from Voight global distributions Inc (Lawrence, KS) and Gallium Indium (GaIn) Eutectic was obtained from ProChem Inc (Rockeford, IL). Ni Wire (99\% pure) and copper sheets were purchased from VWR International (Westchester, PA). Watts nickel plating solution (Watts Ni Pure recipes include $300 \mathrm{~g} / \mathrm{L} \mathrm{NiSO} \cdot 6 \mathrm{H}_{2} \mathrm{O}$, $45 \mathrm{~g} / \mathrm{L}$ each $\mathrm{H}_{3} \mathrm{BO}_{3}$ and $\mathrm{NiCl}_{2} \cdot 6 \mathrm{H}_{2} \mathrm{O}$ ), and $6 \mathrm{M} \mathrm{NaOH}$ were prepared in our laboratory. Panc-1 cells, Dulbecco's Modified Eagle's Medium (DMEM), fetal bovine serum (FBS), 0.25 trypsin/0.53 mM EDTA (trypsin-EDTA), and penicillin-streptomycin antibiotics $(\mathrm{P} / \mathrm{S})$ were purchased from the American Type Culture Collection (ATCC, Rockville, MD). Phosphate buffered saline (PBS) was purchased from Amresco Inc (Salon, OH) and 3-(4, 5-dimethylthiazol-2yl)-2, 5-diphenyl tetrazolium bromide (MTT) cell proliferation assay kits were obtained from Biotium, Inc (CA). $2^{\prime}, 7^{\prime}$-dichlorofluorescein diacetate (DCF-DA) was purchased from EMD chemicals. Acridine orange (AO), ethidium bromide (EB), concentrated nitric acid $\left(\mathrm{HNO}_{3}\right)$, microscope glass slides, and electrical tape were from our laboratory. PI and 
all other biochemicals not otherwise mentioned were all bought from Sigma-Aldrich (St Louis, MO).

\section{Methods}

\section{Fabrication of Ni NWs}

Ni NWs were synthesized by using the electrodeposition syringe method (Figure 1) with modifications. ${ }^{18}$ A custom-made setting of $1.5 \mathrm{~V}$ (AA) battery holder and systems for the electrolysis of the nickel was used to prepare the Ni NWs. In brief, the synthesis of Ni NWs was accomplished by using an alumina $\left(\mathrm{Al}_{2} \mathrm{O}_{3}\right)$ anodisc membrane as a template. One side of the alumina anodisc membrane was coated with the gallium-indium (GaIn) alloy which creates a conductive surface against a copper electrode. A pure Ni electrode was also added to the electrolytes. The synthesis of the Ni NWs takes place in a Ni plating solution. Following this, the alumina disc was removed and taped with its shiny side up onto a glass slide. Concentrated $\mathrm{HNO}_{3}$ was used to complete removal of all the GaIn from the alumina anodisc. The alumina anodisc was then washed with deionized water six times. Sodium hydroxide $(6 \mathrm{M} \mathrm{NaOH})$ was used to dissolve the alumina disc which had Ni NWs on its pore. The polystyrene support ring was then separated from the alumina filter on a beaker and discarded. Using a strong magnet, the Ni NWs were collected and the $\mathrm{NaOH}$ was poured off. The Ni NWs were washed at least 5 times to ensure the complete removal of the trace elements of $\mathrm{NaOH}$. Finally, the Ni NWs were suspended in double distilled water $\left(\mathrm{ddH}_{2} 0\right)$ in a $320 \mathrm{~mL}$ vial for analysis or extended storage. Structural and morphological characterization of synthesized Ni NWs was performed by using energy dispersive X-ray analysis (EDAX), UV-Vis spectroscopy, magnetization study, scanning electron microscopy (SEM), and transmission electron microscopy (TEM).

\section{Transmission electron microscopy}

The sample of Ni NWs was collected and placed onto a formvar coated 200 mesh copper grid by evaporating a drop of $\mathrm{ddH}_{2} \mathrm{O}$ containing well dispersed $\mathrm{Ni} \mathrm{NWs}$ and photographed with transmission electron microscope (JEOL JEM1200 ${ }^{\circledR}$ EX-II) with AMT CCD imaging system at $120 \mathrm{kV}$.

\section{Scanning electron microscopy}

The synthesized Ni NWs were suspended in $\mathrm{ddH}_{2} 0$. A few drops of suspension were then put on a p-Si substrate using silver paint and allowed to dry overnight. A Philips XL $30^{\circledR}$ environmental scanning electron microscope (ESEM) was used to take the micrograph of the fabricated Ni NWs.

\section{EDAX}

Purity and elemental chemical analysis of prepared Ni NWs was performed by using EDAX attached with a Philips XL $30^{\circledR}$ ESEM.

\section{Magnetization properties}

Powerful magnets were used to characterize the magnetization properties of Ni NWs by suspending the Ni NWs in $\mathrm{ddH}_{2} \mathrm{O}$ and photographing them with a 5.1 megapixel Nikon digital camera.

\section{UV-VIS spectroscopy}

The UV-VIS spectrum of Ni NWs was monitored using a Fisher Scientific NanoDrop 2000 spectrophotometer while suspended in $\mathrm{ddH}_{2} \mathrm{O}$ with a baseline correction at $750 \mathrm{~nm}$.

\section{Concentrations of the fabricated Ni NWs working suspensions}

Prepared Ni NWs were washed several times until a neutral $\mathrm{pH}$ (7.00) was obtained. These NWs were sonicated 10 minutes for well dispersion and resuspended in fresh DMEM cell culture medium to a final concentration of approximately $3 \mathrm{NWs}$ for each cell (3:1). The concentration of the Ni NWs in aqueous suspension was measured by a haemacytometer (Hausser Scientific Bright Line) and observed with a $20 \times$ objective using

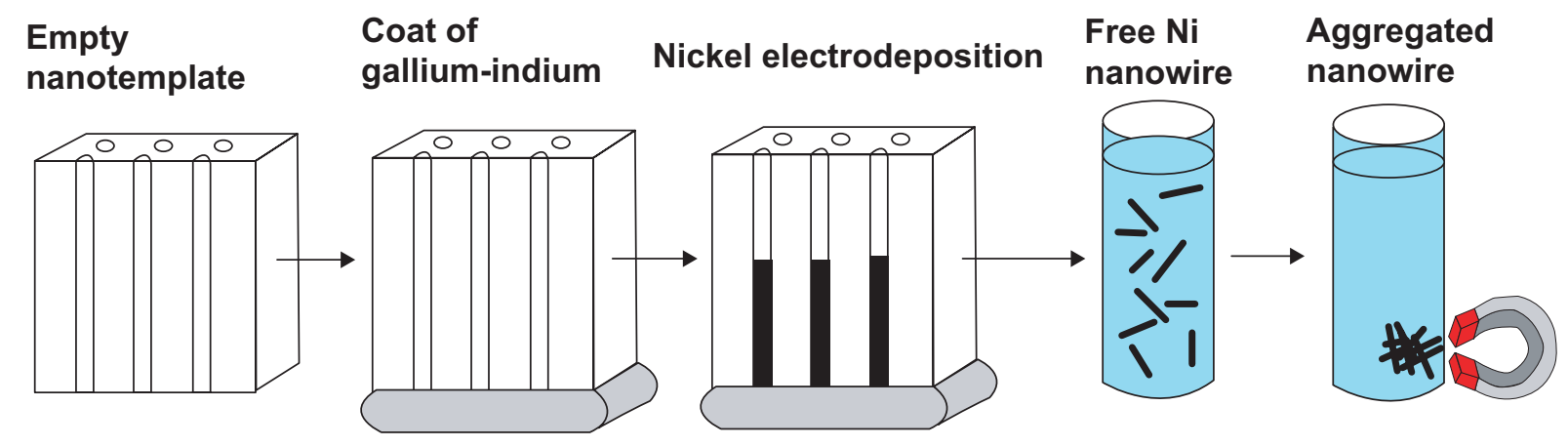

Figure I Schematic of Ni NWs fabrication procedure. 
a Zeiss inverted phase contrast 40 CFL microscope with Spot Basic camera software. In this study, the Ni NWs and cell ratio was kept very low (3) to guarantee a low cytotoxicity. ${ }^{15}$

\section{Cell culture and assessment of growth}

The Panc- 1 cells with a concentration ranging between $2 \times 10^{4}$ and $2 \times 10^{5}$ cells $/ \mathrm{cm}^{2}$ were cultured in DMEM supplemented with $10 \%$ heat-inactivated FBS and $100 \mathrm{IU} / \mathrm{mL}$ P-S in a $75 \mathrm{~cm}^{2}$ flask. Cells were incubated at $\mathrm{pH} 7.4$ at $37^{\circ} \mathrm{C}$ in a humidified chamber equilibrated with $95 \%$ air or $5 \% \mathrm{CO}_{2}$. The medium was changed every 3 to 4 days and subculture was routinely performed at a $60 \%$ to $70 \%$ confluence using trypsin-EDTA to detach the cells. For all experiments, cells were seeded to provide experimental stages, $70 \%$ to $80 \%$ confluence in 6-, 24-, 96-wells plates grown for 24 hours. After 24 hours, cells were washed with pre-warmed $1 \times \mathrm{PBS}(\mathrm{pH} 7.4)$ to remove all traces of serum. The cells were then grown in starvation medium (SMEM) (a normal MEM medium but with reduced 2\% FBS) for an additional 24 hours before all experiments. Representative Panc-1 cultures were harvested and monitored for cell numbers by counting cell suspensions with hemacytometer.

\section{Morphological analysis after internalization of Ni NWs and MTT metabolic activity study by phase contrast microscopy (PCM)}

Intracellular uptake of Ni NWs through endocytosis and metabolic activity of Panc-1 cells was observed by Zeiss Axiovert $40 \mathrm{CFL}^{\circledR}$ inverted phase contrast microscope in a configuration with transmitted light of $12 \mathrm{~V}, 35 \mathrm{~W}$ halogen lamp and $40 \times / 0.50$ LD A-Plan.

\section{Apoptosis assay by fluorescence microscopy}

Ni NW-induced apoptosis in Panc-1 was investigated by staining the cells with a combination of fluorescent $\mathrm{AO}$ and EB dyes followed by BD Biosciences protocol with modifications. In short, cells were grown on an $18 \mathrm{~mm} \times 18 \mathrm{~mm}$ cover slip in a 6 -well plate up to $70 \%$ to $80 \%$ confluency $\left(1 \times 10^{6}\right.$ cells/well). Cells were treated with Ni NWs suspensions of various concentrations, which are mentioned separately in figure captions for the corresponding experiment. After the appropriate incubation time was completed, cells were washed with $1 \times$ PBS and stained with $200 \mu \mathrm{L}$ of dye mixture containing $15 \mathrm{mg} / \mathrm{mL} \mathrm{AO}$ and $50 \mathrm{mg} / \mathrm{mL}$ of $\mathrm{EB}$ mixed in $1 \times$ PBS for 2 to 3 minutes then were washed with PBS twice. The cover slips were then transferred to a sterile glass slide, turned upside down and observed and photographed immediately under fluorescence microscope using 3 to 5 randomly (Nikon Eclipse $90 \mathrm{i}^{\circledR}$ equipped with a digital camera DS-Fi1, NIS-Elements ${ }^{\circledR}$ version 3.1 software at a magnification of 40 times) selected fields of each sample. For negative control images, cells without any treatment stained with AO and EB were used. For apoptosis percentage determination, photographic images were used to determine the number of live cells that were fluorescence green and the number of apoptotic cells that were fluorescence red-orange. Approximately 200 to 300 cells per treatment were counted for apoptosis-related statistical analysis.

\section{MTT assay}

The viability of the Panc-1 cells was quantified by using MTT assay with slight modifications. In brief, Panc-1 cells were plated onto a flat-bottomed 96-well cell culture plate (Bioexpress) for 48 hours (until they had grown approximately 10,000 cells/well) using DMEM with 10\% FBS and $1 \% \mathrm{P} / \mathrm{S}$ solution in the dark at $37^{\circ} \mathrm{C}$ incubator with $5 \% \mathrm{CO}_{2}$. The Ni NWs treatment was done with concentrations of $0 \mu \mathrm{L}$ (without Ni NWs as control), $10 \mu \mathrm{L}, 50 \mu \mathrm{L}, 100 \mu \mathrm{L}$, and $200 \mu \mathrm{L}$ and allowed to grow for 24 hours. After the appropriate treatment time, the cell culture medium was discarded, and to remove the traces of uninternalized $\mathrm{Ni}$ NWs, each well was washed 3 times with $1 \times$ PBS. After that, $10 \mu \mathrm{L}$ MTT solution was added to each well of the 96-well plates containing Panc-1 cells, mixed by shaking gently on an orbital shaker for 5 minutes, and incubated for 4 hours at $37^{\circ} \mathrm{C}$. Then the medium was removed and $200 \mu \mathrm{L}$ of dimethyl sulfoxide was added into each well and pipetted up and down several times to dissolve the formazan created by the MTT. Absorbance was measured in a 96 well-microplate reader (BIOTEK $\mu$ Quant ${ }^{\circledR}$ ) at $570 \mathrm{~nm}$ with reference $O D$ at $630 \mathrm{~nm}$. Each treatment was replicated in triplicate and the mean value was used to calculate the data. For qualitative analysis, phase contrast images were taken randomly.

\section{Cell cycle analysis using flow cytometry (FC)}

To analyze cell cycle with FC of Ni NW-induced apoptotic Panc-1 cells, PI staining method was used with optimization and modification. Various concentrations of Ni NWs were used to induce apoptosis in Panc-1 cells using the methodology described in the apoptosis induction section above. A negative control was prepared by incubating cells without Ni NWs. After the incubation period of the appropriate treatment was completed, Panc-1 cells were harvested using TE and washed 3 times using pre-warmed $1 \times$ PBS to remove the trace amount of the apoptosis inducing agent. The density of the cells was adjusted to $\sim 1 \times 10^{6}$ cells $/ \mathrm{mL}$ 
in PBS. To stain the Panc-1 cells on FC, for each assay of $1 \mathrm{~mL}$ cell suspension (in PBS), $0.5 \mu \mathrm{L}$ of PI $(1.5 \mu \mathrm{M})$ was added to the FC tubes. The stained cell suspension was then transferred and kept on ice to incubate for 30 minutes. Following this incubation period, the stained cells were quantified and data were produced by a Becton Dickinson (BD) FACS Calibur ${ }^{\circledR}$ flow cytometer. The Cell Quest Pro ${ }^{\circledR}$ software was then used to analyze the data. Approximately 10,000 events were quantified using a FACS Calibur ${ }^{\circledR}$ with an excitation wavelength of $536 \mathrm{~nm}$ and an emission wavelength of $617 \mathrm{~nm}$.

\section{Detection and quantification of intracellular ROS production}

To determine the intracellular ROS generation, the Panc- 1 cells were labeled with the fluorescent DCF-DA probe and measured with a BD FACSCalibur ${ }^{\circledR}$ flow cytometer. Panc-1 cells were seeded in 6 -well plates up to $70 \%-80 \%$ confluence. The cell culture media was then replaced with SMEM 24 hours before appropriate Ni NW-treatment to induce apoptosis. After 24 hours of incubation, cells were incubated with $10 \mu \mathrm{M}$ of DCF-DA at $37^{\circ} \mathrm{C}$ for 30 minutes. Following this, the cells were trypsinized, centrifuged, then the pellets were collected in FC tubes and resuspended in $1 \mathrm{~mL}$ of $1 \times \mathrm{PBS}$ and analyzed on BD FACSCalibur $^{\circledR}$ with an excitation wavelength of $488 \mathrm{~nm}$ and an emission wavelength of $530 \mathrm{~nm}$. For data analyses the Cell Quest ${ }^{\circledR}$ Pro software was used.

\section{Statistical analysis}

All results were expressed as mean $\pm \mathrm{SE}$. The significance of difference was evaluated with one way analysis of variance (ANOVA). A probability level of $P<0.05$ was considered statistically significant different from the control.

\section{Results}

\section{Structural and morphological characterization of synthesized Ni NWs}

In the EDAX spectrum (Figure 2A) of the Ni NWs, the presence of nickel with a very tiny amount of oxygen and iron was found. Weight percentages by element are nickel 42.33 , oxygen 4.58 , iron 0.85 , and silicon 52.24. This small portion of oxygen is due to the Ni NWs being exposed to air in the environment. The very minute level of iron resulted from pre-existing contamination in the ESEM and EDAX
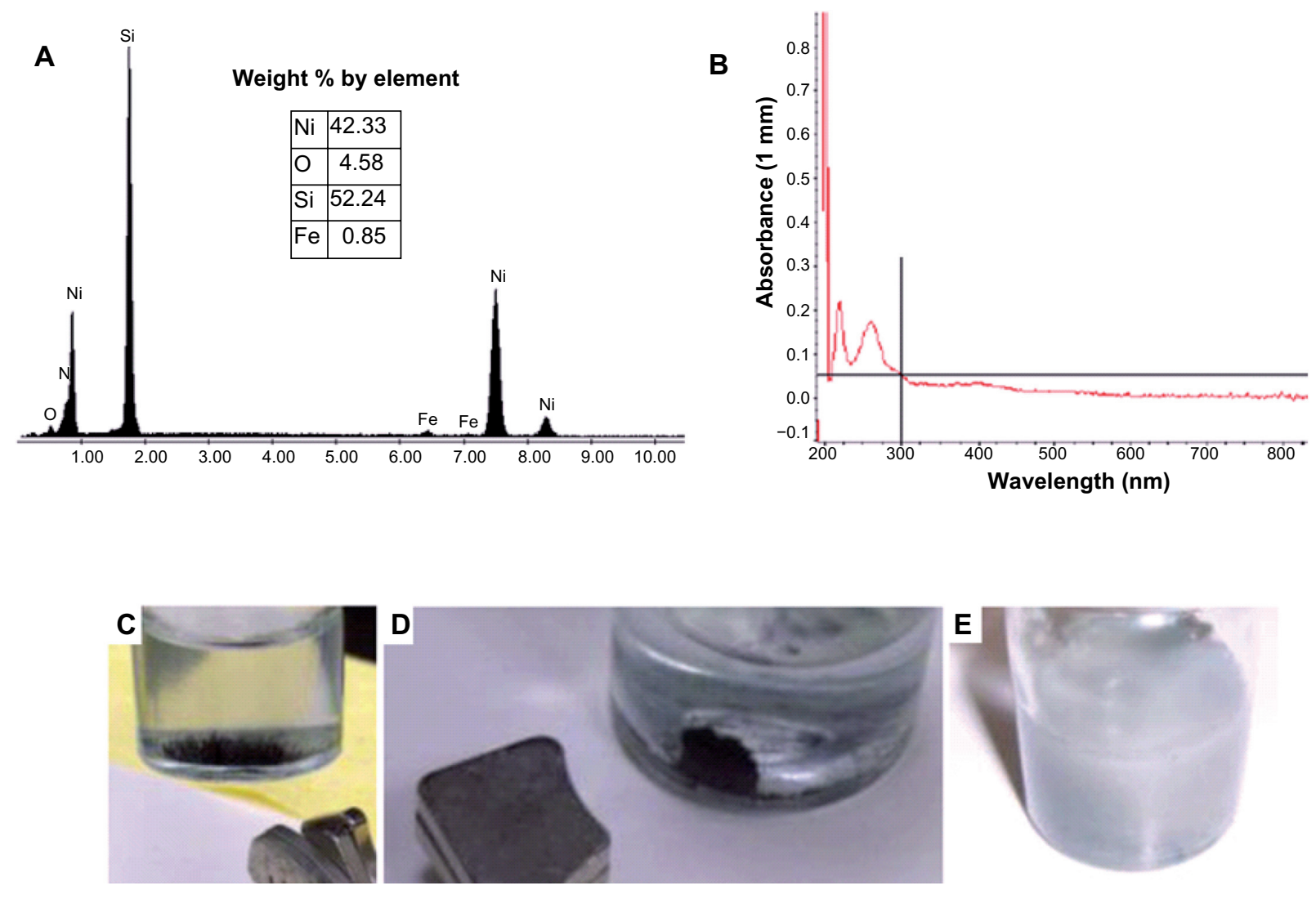

Figure 2 Characterization of fabricated Ni NWs. (A) Energy dispersive X-ray analysis spectrum of Ni NWs for elemental analysis; (B) UV-Vis spectrum of Ni NWs suspended in $\mathrm{ddH}_{2} \mathrm{O}$ at neutral pH (7.00); (C) vertically aligned magnetic Ni NWs on the bottom of a beaker; (D) illustration of magnetic properties of Ni NWs; (E) Ni NWs suspended in aqueous media. 

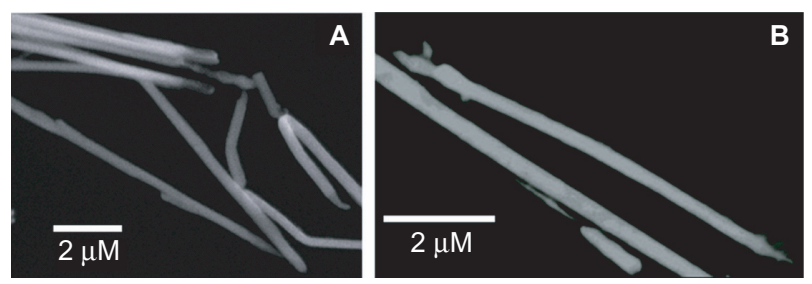

Figure 3 Electron micrograph of Ni NWs. (A) SEM of Ni NWs; (B) TEM of Ni NWs. Abbreviations: SEM, scanning electron microscopy; TEM, transmission electron microscopy.

chamber. The silicon peak is justified by the silicon wafer chip, which was used to augment visibility of the Ni NWs in the SEM and EDAX by increasing contrast with a low background. Therefore, the EDAX data analysis revealed that the resultant Ni NWs were pure Ni metal.
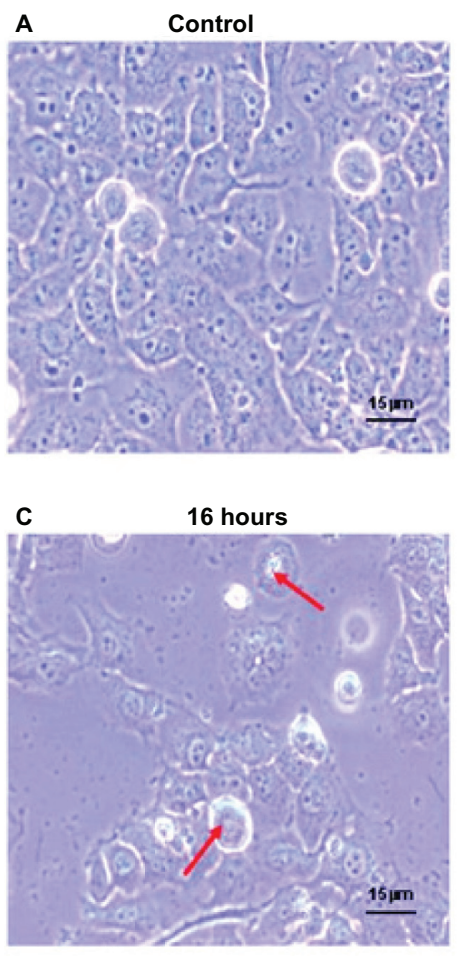

E

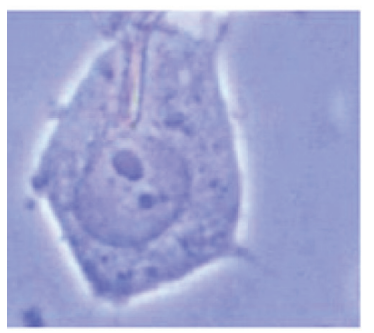

$\mathbf{F}$

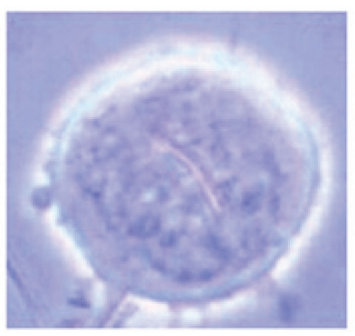

The UV-VIS spectrum (Figure 2B) of the Ni NWs suspension was evaluated using the Fisher Scientific NanoDrop 2000 spectrophotometer. The absorption spectrum displayed the optical properties and characteristic broadband at $264 \mathrm{~nm}$ with an absorbance of 0.18 . The absorption spectrum has two peaks which may be attributed to the surface plasmon excitation. ${ }^{19}$

Figures $2 \mathrm{C}$ and 2D illustrate the magnetic properties of $\mathrm{Ni}$ $\mathrm{NWs}$ in aqueous solution regulated with strong magnets. More specifically, in Figure 2C, Ni NWs are aggregated at the bottom of the vial in a vertical magnetic field and in Figure 2D, Ni NWs are collected on the sidewall of the vial illustrated by the black aggregate. Figure 2E depicts the Ni NWs in aqueous suspension which was used for apoptosis experiments in Panc-1.
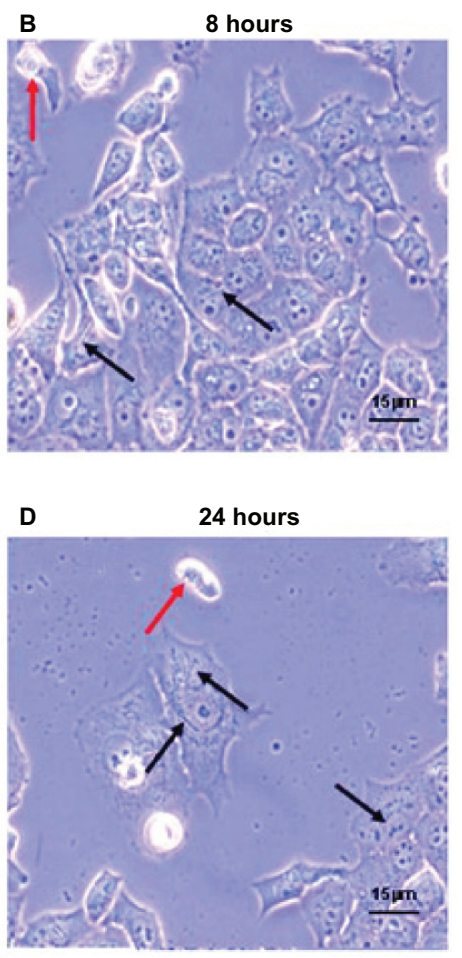

G

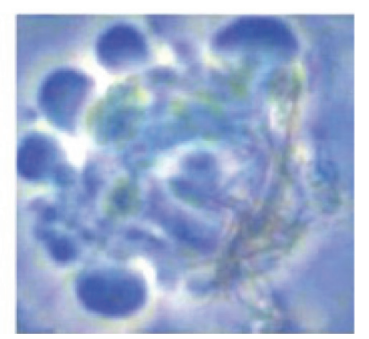

Figure 4 Morphological characteristics of Panc-I cells which were treated with $200 \mu \mathrm{l}$ of Ni NWs suspension for 0 (control), 8 , I6, and 24 hours and visualized with phase contrast microscope (magnification $40 \times$ ). Increasing detachment of cells from the well surface is seen with increasing exposure time. (A) Adherent cells without Ni NWs (control); (B) adherent (black arrows) and suspended (red arrows) cells with internalized Ni NWs after 8 hours; (C) adherent and a greater number of suspended cells with internalized Ni NWs after 16 hours; (D) Adherent and suspended cells with internalized Ni NWs after 24 hours. (E-G) represent more specific cellular morphological changes. (E) Uptake of Ni NWs by endocytosis to the cell cytoplasm; (F) internalization of Ni NWs to the cell nucleus followed by cell shrinkage, round shaped, and initiation of membrane blebbing; (G) apoptotic body formation through final membrane blebbing. 


\section{Diameter and length of the fabricated Ni NWs}

SEM and TEM were used to determine the length and diameter of Ni NWs. Figure 3A shows the representative SEM of the top view of Ni NWs and Figure 3B portrays a micrograph of a TEM view of the fabricated Ni NWs. The diameter of the fabricated Ni NWs ranged from $200 \mathrm{~nm}$ to $225 \mathrm{~nm}$ with the average of $215 \mathrm{~nm}$. The average length of the Ni NWs was found to be $6.5 \mu \mathrm{m}$.

\section{PCM for morphology and biocompatibility of internalized Ni NWs in Panc-I cells}

Compared with the control, as depicted by Figures 4A-D, there was increasing detachment of cells from the well surface in addition to internalization of Ni NWs with increasing exposure time. Apoptotic morphological changes are portrayed in Figures 4E-G showing cell shrinkage, round apoptotic bodies, and membrane blebbing in PCM (magnification 40×). The biocompatibility of the fabricated Ni NWs has been investigated in Panc-1 cell lines. It was determined that $\mathrm{Ni}$ NWs (diameter $\sim 215 \mathrm{~nm}$ and length up to $7 \mu \mathrm{m}$ ) can easily be internalized by Panc-1 cells. The Ni NWs are indicated by arrows in the figures. Adherent cells with internalized $\mathrm{Ni}$
NWs are designated by black arrows and suspended cells with internalized Ni NWs are indicated by red arrows.

\section{Fluorescent pictures of Panc-I cells after Ni NW-induction of apoptosis}

Figures 5A-D show fluorescence pictures with advancing exposure time to the Ni NWs suspension compared with the control. With increasing exposure time to Ni NWs, fewer Panc-1 cells survived. Cells were stained with AO and EB dyes. Viable Panc-1 cells displayed green nuclear fluorescence and apoptotic cells exhibited a red nucleus due to DNA binding capacity of EB. The bar graph in Figure 6 portrays a direct relationship between concentration of $\mathrm{Ni}$ NWs and extent of apoptosis in Panc-1 cells.

\section{MTT assay for cellular proliferation, viability, and metabolic activity study}

Figure 7 shows qualitative morphological study of PCM on Panc-1 cell viability and proliferation. It is evident that the morphology of Panc-1 cells treated with Ni NWs differs from the controls (ie, without treatment). As shown in Figure 7, formazon grew on live or metabolically active cells as depicted by the deep purple cell nucleus. However, dead

B
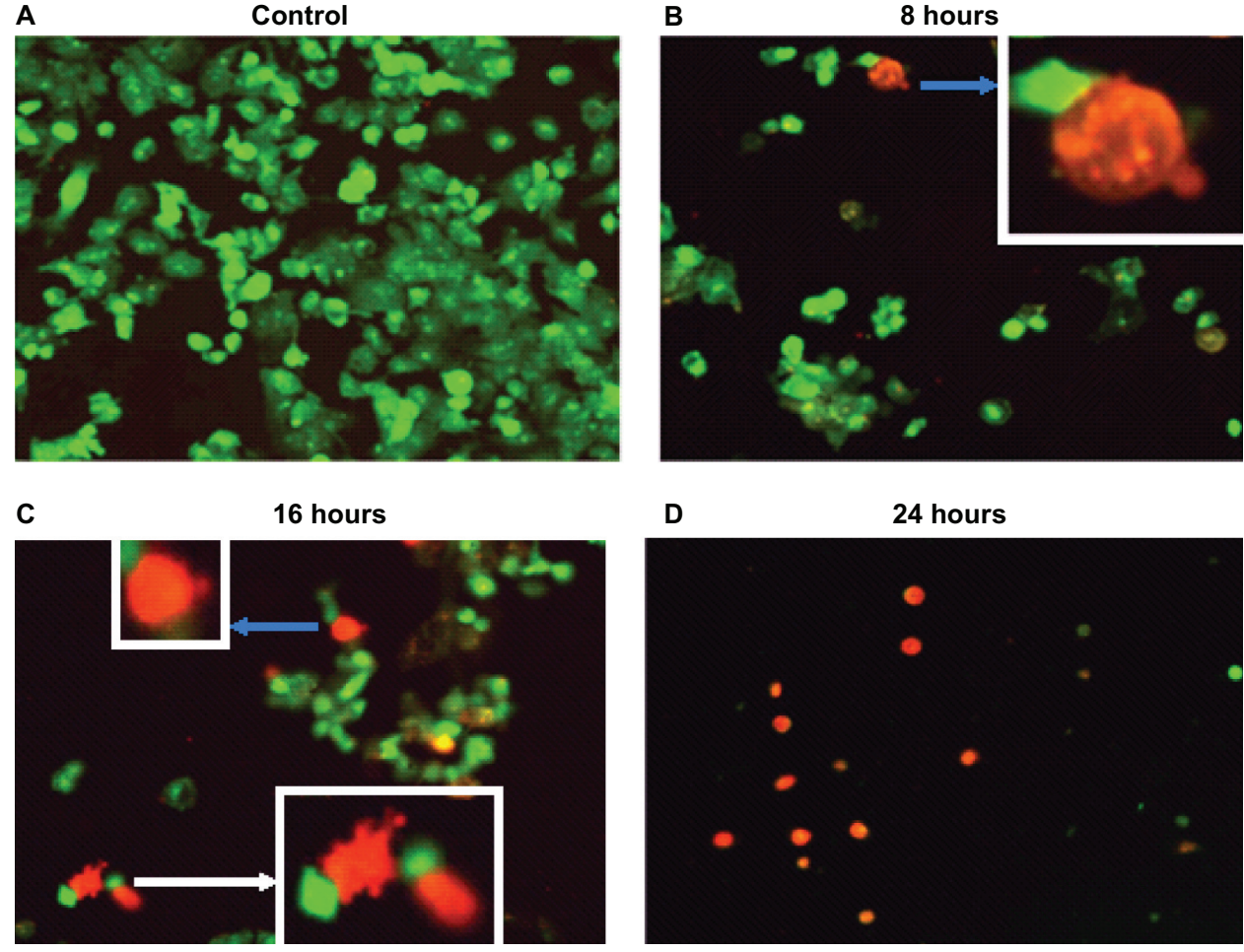

D

24 hours

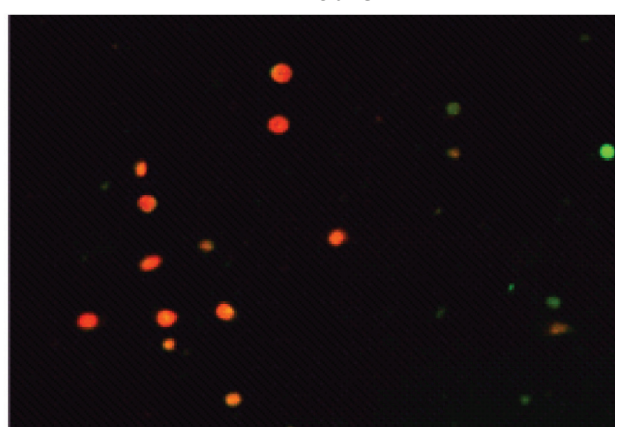

Figure 5 Panc-I cells treated with Ni NWs which potentiates apoptosis. Cells were treated with $200 \mu \mathrm{L} \mathrm{Ni} \mathrm{NWs} \mathrm{for} \mathrm{8,} \mathrm{16,} \mathrm{and} \mathrm{24} \mathrm{hours} \mathrm{and} \mathrm{stained} \mathrm{with} \mathrm{ethidium} \mathrm{bromide}$ (EB) and acridine orange (AO) and were visualized and photographed immediately with fluorescence microscope. (A) Control; (B) 8 hours after treatment - blue arrow indicates initial membrane blebbing of the apoptosed cells; (C) 16 hours after treatment - the white arrow indicates late membrane blebbing; (D) 24 hours after treatment. Cells were stained with $\mathrm{AO}$ and EB where viable cells were green nuclear fluorescence and apoptotic cells exhibit a red nucleus. 


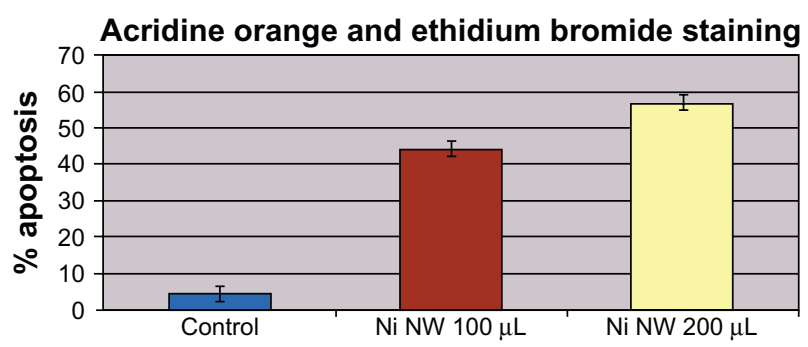

Figure 6 Effects of concentration of Ni NWs on apoptosis of Panc-I cells after 24-hour exposure.

cells did not grow formazon and therefore the color was not changed. Black and white arrows in Figure 7 indicate dead and live cells respectively. For quantitative study of cell viability and proliferation, we used MTT assay and analyzed the results with a microplate reader. The bar graph in Figure 8 displays the dose and time-dependent effects of Ni NWs on the proliferation of Panc 1 cells. In this figure, the percentages of viable cells with different concentrations of NI NWs were shown and compared with the control in which the cells are approximately $100 \%$ viable.

\section{Apoptotic cell cycle analysis through FC}

Cell cycle analysis of Ni NW-induced apoptosis in Panc-1 cells was shown in Figure 9. The cells were incubated with Ni NWs (Figure 9B) for 24 hours, stained with PI, and analyzed by FC (BDFACS Calibur ${ }^{\mathbb{R}}$ ). These results were compared with the control. The increase of sub- $\mathrm{G}_{1}$ phase accumulation is the biomarker of apoptosis. ${ }^{20}$ This is visualized in Figure 9 where the percentage of the sub- $\mathrm{G}_{1}$ peak is exclusively increased, which is an indicator of apoptosis. FC analysis showed that the Ni NW-induced
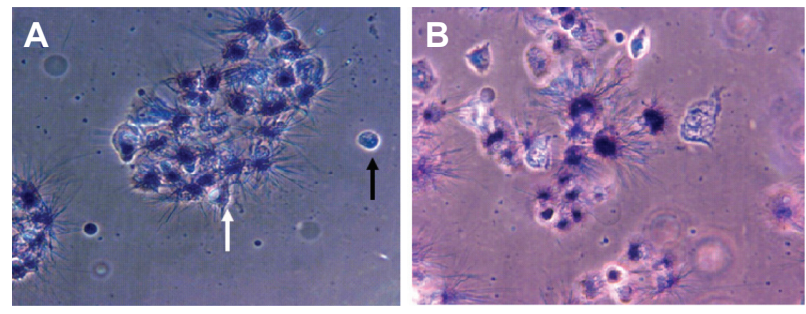

Figure 7 Qualitative morphological study of apoptosis by MTT assay. Phase contrast pictures of formazon in Ni NWs treated Panc-I cells where $(\mathbf{A})$ control, ie, untreated cells and (B) treated cells. Viable cells exhibited a deep purple cell nucleus with rod shaped formazon whereas apoptotic cells lacked formazon growth because of the lack of mitochondrial reductase and thus did not change in color. Dead cells are indicated by black arrows and live cells are identified by white arrows.

Panc-1 cell proliferation was suppressed and cell cycle arrest occurred in $\mathrm{G}_{0} / \mathrm{G}_{1}$ phase. Thus, our study suggested that $\mathrm{Ni}$ NWs modulate cellular response through apoptosis effectors involved in cell cycle. In the cell cycle, 2 check points $G_{1}$ and $\mathrm{G}_{2}$ play a critical role in the regulation of cells going from $\mathrm{S}$ and $\mathrm{M}$ phases such that apoptotic or dead cells stop DNA replication at $\mathrm{G}_{1}$ or $\mathrm{G}_{2} \cdot{ }^{17}$ In our study involving flow cytometric DNA content analysis, cell cycle was terminated in the sub- $\mathrm{G}_{1}$ phase. These data establish that the Panc-1 cell cycle was halted as a result of exposure to Ni NWs which clearly demonstrates apoptosis.

\section{Quantification of endogeneous ROS generation with DCF fluorescence of FC}

ROS has major functions in a series of biological responses and diverse signaling pathways including apoptosis. ${ }^{21}$ ROS production by Ni NWs was measured by FC. Figure 10 shows Ni NWs-induced ROS generation of Panc-1 cells. Panc-1 cells were treated with different concentrations of

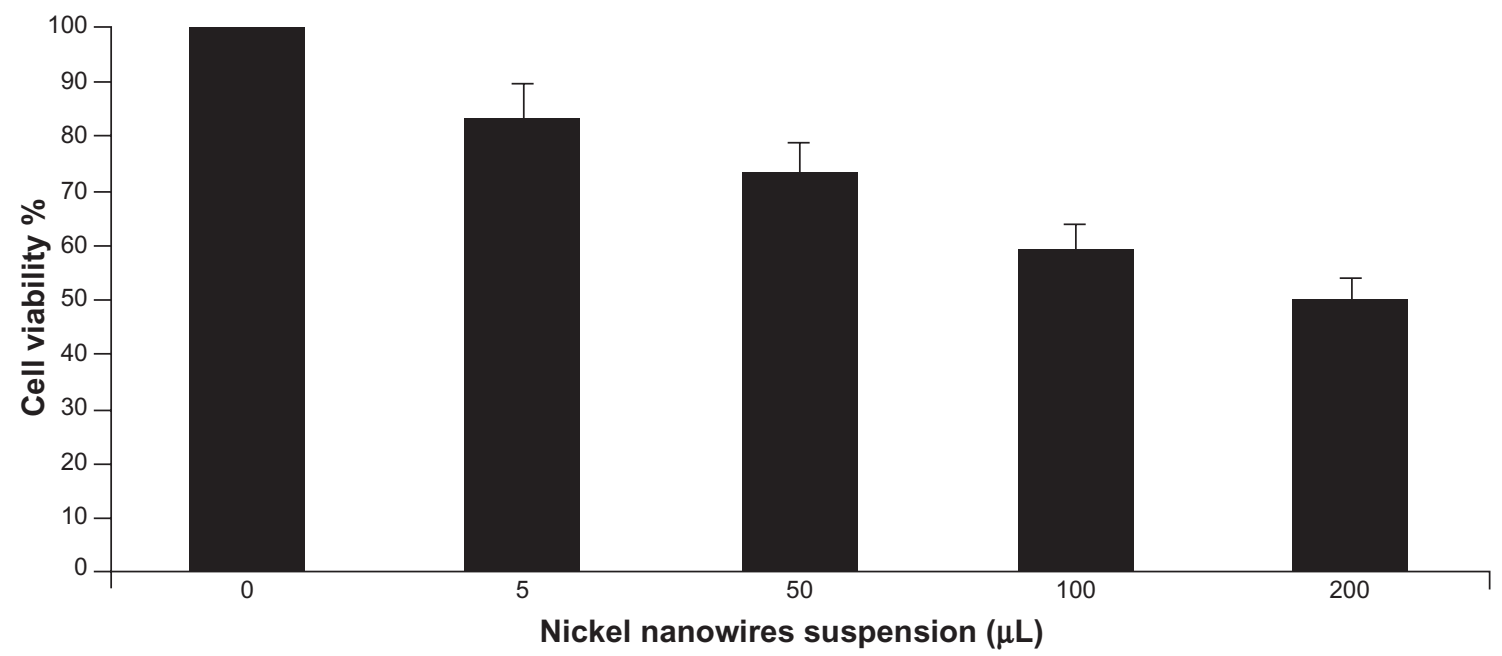

Figure 8 Quantitative 3-(4,5-dimethylthiazol-2yl)-2,5-diphenyl tetrazolium bromide (MTT) assay of cell viability and proliferation. Cell viability assay shows the effect of Ni NWs as concentration dependent reduction of viability in Panc-I cells. 

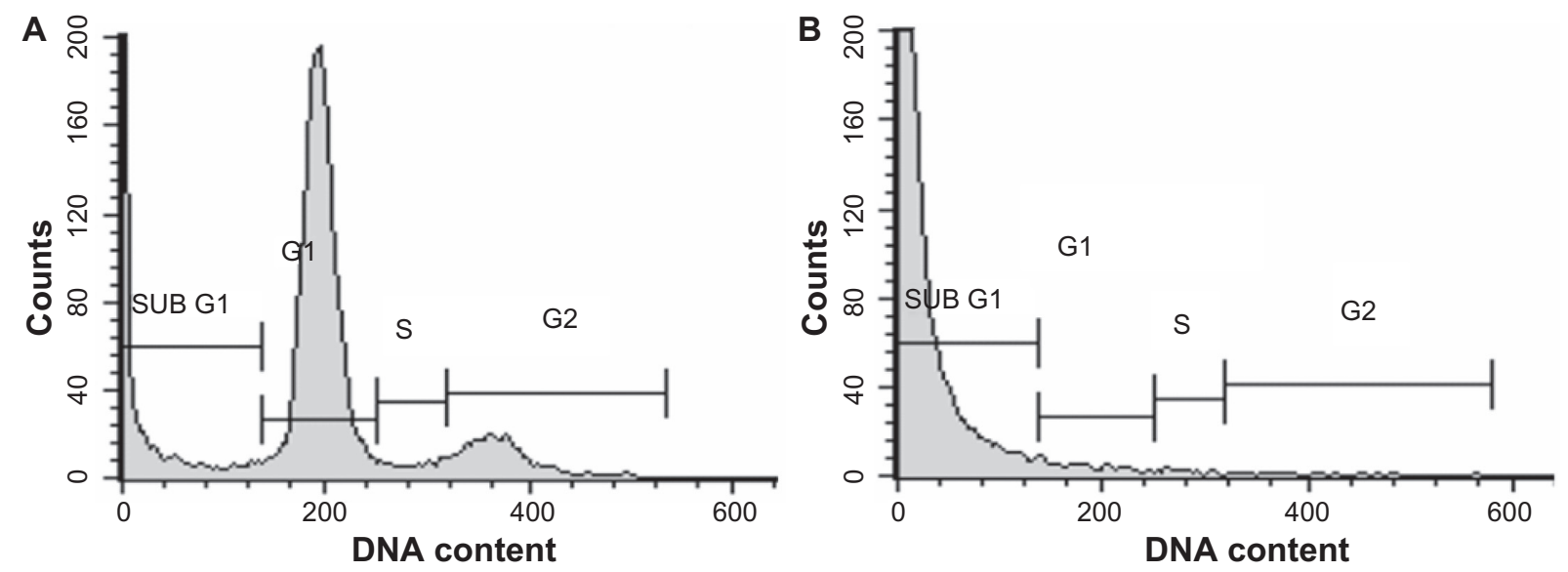

Figure 9 Cell cycle analysis after induction of apoptosis in Panc-I cells by Ni NWs. The cells were incubated with Ni NWs for 24 hours, stained with PI and analyzed by Cell Quest Pro ${ }^{\circledR}$ on FC (BD-FACS Calibur ${ }^{\circledR}$ ). A portrays untreated cells with all phases of the cell cycle, whereas B shows an exclusively increased sub-G, peak with Ni NWs treatment.

Ni NWs over 24 hours. Cells without treatment were used as the control (Figure 10A), cells treated with $\mathrm{H}_{2} \mathrm{O}_{2}(0.3 \%)$ were used as the positive control (Figure 10B), and Ni NWtreated cells (Figure 10C). Figure 10D (representative overlay histograms of Figures 10A-C) shows increases of
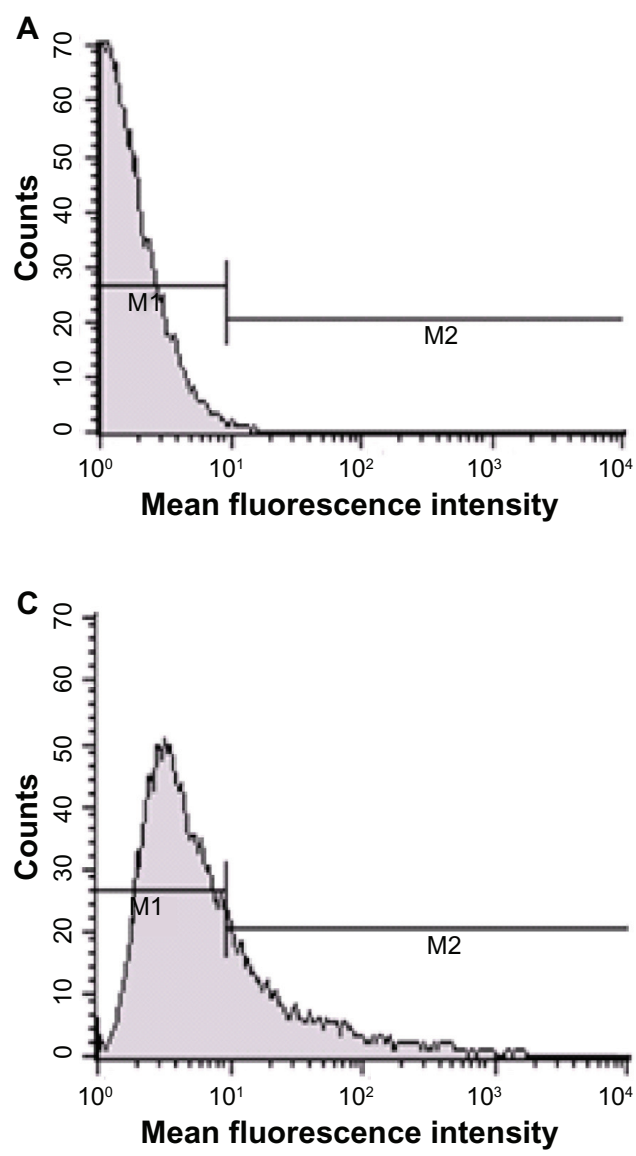

mean fluorescence intensity (oxidant production) as right shifts on the $\mathrm{x}$-axis.

The bar graph in Figure 11 represents the concentration dependent ROS generation which is quantified using DCF-DA by FC, where the $\mathrm{x}$-axis portrays the concentration of Ni NWs
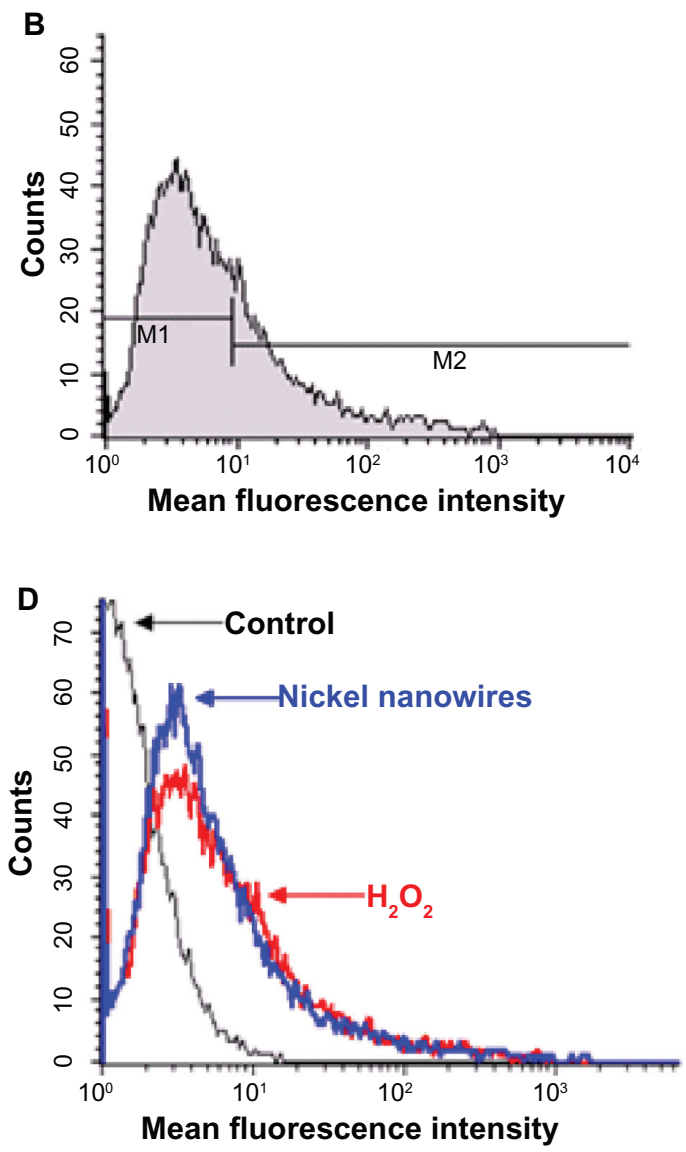

Figure 10 Ni NWs-induced reactive oxygen species generation in Panc-I cells were measured by flow cytometry. The fluorescence intensities of I0,000 cells were analyzed by BD-FACS Calibur ${ }^{\circledast}$. Representative histograms show cell number on the $y$-axis and increases of fluorescence (oxidant production) as right shifts on the $x$-axis (mean fluorescence intensity). (A) Cells without treatment; (B) cells treated with $0.3 \% \mathrm{H}_{2} \mathrm{O}_{2}$; (C) Ni NWs treated cells; (D) overlay histogram of $\mathbf{A}-\mathbf{C}$. 


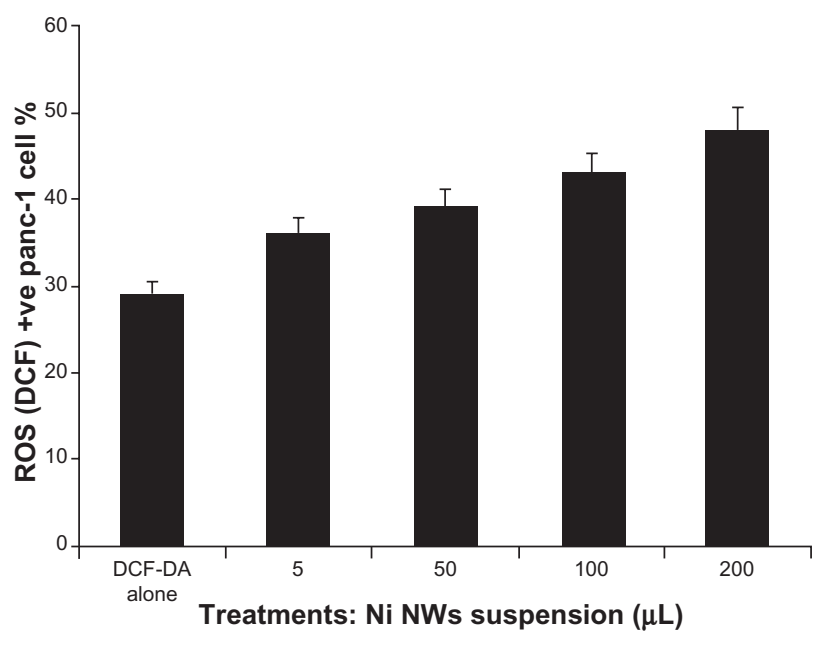

Figure I I Quantification of reactive oxygen species (ROS) generation on Ni NWinduced Panc-I cells stained with DCF-DA dye and measured by flow cytometry. This bar graph represents the concentration dependent ROS generation where the $\mathrm{x}$-axis represents the concentration of Ni NWs and the $y$-axis denotes the percentage of ROS (DCF)-positive Panc-I cells.

and the $y$-axis denotes the percentage of ROS (DCF) positive Panc-1 cells. We further examined the correlation between the rate of apoptosis and the ROS generation by Ni NW-treated cells. A significant positive correlation was observed with a correlation coefficient of 0.99373 . These results clearly demonstrated that Ni NWs induced ROS generation and ultimately apoptotic cell death.

\section{Discussion}

Structural and morphological characterization of synthesized Ni NWs was determined by using EDAX, UV-Vis, magnetization study, SEM, and TEM. From these analyses we gained the compositions, diameter, length, and characteristics of the Ni NWs and came to the conclusion that Ni NWs prepared in our laboratory were pure Ni metal.

In this study, by using several standard apoptotic analysis strategies, we demonstrated that Ni NWs induced apoptosis through ROS generation. First, we microscopically observed morphological changes showing the evidence of Ni NWs induced apoptosis in Panc-1 cells through typical apoptosis features such as cell shrinkage, cell membrane blebbing, detachment from surface, nuclear condensation and apoptotic round-shape body formation. Secondly, we illustrated that Ni NWs enhanced cell death in Panc-1 cells as proven by an increased sub- $\mathrm{G}_{1}$ peak percentage of cell cycle arrest which mediates the increased apoptotic cell percentage in DNA FC. Third, since nanomaterials do not have the same properties as bulk materials, ${ }^{13}$ in the global cytotoxicity perspective, cytotoxicity of nanomaterials in cancer cells should be distinguished from cell specific or cell receptor-specific toxicity. ${ }^{21}$ To investigate the mechanism of Ni NWs-induced cytotoxicity, we examined the mitochondrial activity assay in Panc-1 cells both quantitatively and qualitatively. The viability result of MTT implies that the cytotoxic effect of Ni NWs is related to programmed cell death. In our study, it was found that the viability of the Panc-1 cancer cells decreased as the concentration of the Ni NWs and time of exposure increased.

ROS such as $\mathrm{H}_{2} \mathrm{O}_{2}$ have been considered as cytotoxic byproducts of cellular metabolism, and the overproduction of ROS in cells may enhance cell death. ${ }^{22,23}$ We next examined whether Ni NWs have the ability to generate endogeneous ROS in Panc-1 cells. For this reason, cells treated with Ni NWs were stained with ROS specific DCF-DA dye which is specific for $\mathrm{H}_{2} \mathrm{O}_{2}$. In Figure 10D, rightward shift of the peaks indicate that cellular ROS were strongly induced by $\mathrm{Ni}$ NWs through $\mathrm{H}_{2} \mathrm{O}_{2}$ accumulation, suggesting that Ni NWs function as an apoptotic agent in cancer cells. Furthermore, significant cellular ROS accumulation was detected in Ni NW-treated Panc-1 cells which exert anti-cancer effects through $\mathrm{H}_{2} \mathrm{O}_{2}$ generation.

Our results point to a positive correlation between the rate of apoptosis and the ROS generation induced by Ni NWs with a concentration dependent fashion. These findings are also confirmed by cellular and nuclear morphological changes, loss of viability in concentration-dependent manner (MTT assay), increase in PI uptake, and resultant increase in sub- $\mathrm{G}_{1}$ peak in DNA content analysis of FC. We have shown that without any specific surface functionalization, Ni NWs were able to induce apoptosis in certain cancer cell lines such as Panc-1.

Taken together, our results suggest the molecular mechanisms involved in Ni NWs induced apoptosis through ROS generation in Panc- 1 cells. To the best of our knowledge this is the first report showing Ni NWs-induced ROS mediated apoptotic cell death in Panc-1 cancer cells.

\section{Conclusion}

In summary, the present study indicates that Ni NWs inhibit cell proliferation of Panc-1 cell lines and induce apoptosis by accumulating ROS. More specifically, we have shown that Ni NWs induce oxidative stress, resulting in ROS generation, which in response triggers apoptosis in Panc-1 cells. Overall our data suggest that Ni NWs have the properties of apoptotic agents through cell cycle arrest by diminishing fractions of cell cycle phases excluding the sub-G1 phase, increasing endogenous ROS generation and finally inducing apoptosis in Panc-1 cells. For these reasons, Ni NWs show good potential 
for the treatment of pancreatic cancer selectively and warrant further investigation.

\section{Acknowledgments}

The authors would like to thank Rozina Akter, $\mathrm{PhD}$ candidate, for critically reading the manuscript and Jill L Castleberry, MS candidate for English language copy editing. Md. Zakir Hossain thanks Dr William H Baltosser for his assistance and acknowledges the Graduate Institute of Technology, UALR, for a Graduate Assistantship.

\section{Disclosure}

The authors declare that there are no conflicts of competing interests in relation to this work.

\section{References}

1. National Cancer Institute, US National Institutes of Health, http://www. cancer.gov. and http://www.cancer.gov/cancertopics/types/pancreatic. Accessed at April 18, 2011.

2. Potten C, Wilson J. Apoptosis: the Life and Death of Cells. Cambridge University Press; 2004.

3. Kanduc D, Mittelman A, Serpico R, Sinigaglia E, et al. Cell death: apoptosis versus necrosis (review). Int J Oncol. 2002;21:165-170.

4. Held P, Newick K. Using BioTek's Synergy HT Reader to measure reactive oxygen species (ROS) generation in stimulated cells. Tech Resources. 2008;1-3.

5. Pan J, Chang Q, Wang X, et al. Reactive oxygen species-activated Akt/ ASK1/p38 signaling pathway in nickel compound-induced apoptosis in BEAS 2B cells. Chem Res Toxicol. 2010;23:568-577.

6. Lau ST, Lin ZX, Leung PS. Role of reactive oxygen species in brucein D-mediated p38-mitogen-activated protein kinase and nuclear factor- $\mathrm{\kappa B}$ signalling pathways in human pancreatic adenocarcinoma cells. $\mathrm{Br} J$ Cancer. 2010;102:583-593.

7. Amit TK, Roy M, Bhattacharya RK. Natural products as inducers of apoptosis: implication for cancer therapy and prevention. Curr Sci. 2001;80(11):1387-1396.
8. Zhao J, Bowman L, Zhang X, et al. Metallic nickel nano- and fine particles induce JB6 cell apoptosis through a caspase-8/AIF mediated cytochrome c-independent pathway. J Nanobiotechnology. 2009; 7:2.

9. Sharma M, Sharma PD, Bansal MP, Singh J. Lantadene A-induced apoptosis in human leukemia HL-60 cells. Indian J Pharmacol. 2007; 39:140-144.

10. Available from: http://www.webelements.com/nickel/biology.html. Accessed April 18, 2011.

11. Costa M. Molecular biology of nickel carcinogenesis. Fresenius J Anal Chem. 1998;361:381-385.

12. Choi D, Park J, Kim S, et al. Hyperthermia with magnetic nanowires for inactivating living cells. J Nanosci Nanotechnol. 2008;8:1-5.

13. Guo D, Wu C, Li J, et al. Synergistic effect of functionalized nickel nanoparticles and quercetin on inhibition of the SMMC-7721 cells proliferation. Nanoscale Res Lett. 2009;4:1395-1402.

14. Gao N, Wang H, Yang EH. An experimental study on ferromagnetic nickel nanowires functionalized with antibodies for cell separation. J Nanotechnology. 2010;21(10)105-107.

15. Ward TH, Cummings J, Dean E, et al. Minireview biomarkers of apoptosis. Br J Cancer. 2008;99:841-846.

16. Prina-Mello A, Diao Z, Coey JMD. Internalization of ferromagnetic nanowires by different living cells. J Nanobiotechnology. 2006;4:9.

17. Shiao YH, Lee SH, Kasprzak KS. Cell cycle arrest, apoptosis and p53 expression in nickel (II) acetate-treated Chinese hamster ovary cells. Carcinogenesis. 1998;19:1203-1207.

18. Bentley AK, Farhoud M, Ellis AB, Lisensky GC, Anne-Marie, Crone WC. Template synthesis and magnetic manipulation of nickel nanowires. J Chem Educ. 2005;82:765-768.

19. Sapkal SB, Shelke KF, Shingate BB, Shingare MS. Synthesis, characterization and structural investigations of Ni nanoparticless: an ecofriendly and reusable catalyst for the synthesis of 3, 4-dihydropyrimidine-2(1H)ones via Biginelli reaction. Bull Korean Chem Soc. 2010;31:351.

20. Kezhou C, Chong R, Zengliang Y. Nickel-induced apoptosis and relevant signal transduction pathways in Caenorhabditis elegans. Toxicol Ind Health. 2010;26:249-256.

21. Patra HK, Banerjee S, Chaudhuri U, Lahiri P, Dasgupta AK. Cell selective response to gold nanoparticles. Nanomedicine. 2007;3:111-119.

22. Wang Q, Zheng X, Yang L, et al. Reactive oxygen species-mediated apoptosis contributes to chemosensitization effect of saikosaponins on cisplatin-induced cytotoxicity in cancer cells. $J$ Exp Clin Cancer Res. 2010;29:159.

23. Eruslanov E, Kusmartsev S. Identification of ROS using oxidized DCFDA and flow-cytometry. Methods Mol Biol. 2010;594:57-72.
International Journal of Nanomedicine

\section{Publish your work in this journal}

The International Journal of Nanomedicine is an international, peerreviewed journal focusing on the application of nanotechnology in diagnostics, therapeutics, and drug delivery systems throughou the biomedical field. This journal is indexed on PubMed Central, MedLine, CAS, SciSearch ${ }^{\circledR}$, Current Contents $₫ /$ Clinical Medicine,

\section{Dovepress}

Journal Citation Reports/Science Edition, EMBase, Scopus and the Elsevier Bibliographic databases. The manuscript management system is completely online and includes a very quick and fair peer-review system, which is all easy to use. Visit http://www.dovepress.com/ testimonials.php to read real quotes from published authors. 